\title{
PERCEPCIONES ESTUDIANTILES SOBRE EL PROCESO DE ACREDITACIÓN POR ALTA CALIDAD DEL PROGRAMA DE ADMINISTRACIÓN DE EMPRESAS DE LA UNIVERSIDAD DEL MAGDALENA
}

\author{
STUDENT PERCEPTIONS ABOUT THE ACCREDITATION PROCESS FOR HIGH QUALITY \\ OF BUSINESS ADMINISTRATION PROGRAM FROM UNIVERSITY OF MAGDALENA
}

\author{
Javier Viloria-Escobar*, María Bertel-Narváez ${ }^{* *}$ y Alexander Daza-Corredor ***
}

\begin{abstract}
RESUMEN
El presente artículo tuvo como objetivo explorar las percepciones que tienen los estudiantes del Programa de Administración de Empresas de la Universidad del Magdalena sobre el proceso de acreditación de alta calidad al que está sometido ante el Ministerio de Educación Nacional. Para lo cual se tomó una muestra aleatoria de 100 estudiantes que hacen parte de los diversos semestres académicos y se les aplicó un cuestionario que indagaba por sus apreciaciones sobre el proceso. Los principales resultados permiten sostener que los estudiantes tienen un gran sentido de pertenencia con el Programa y consideran que se merece la acreditación por parte del Ministerio y, que además este certificado se convierte en algo positivo para sus carreras profesionales. Se concluye que, a pesar de la evidente aceptación de las condiciones de calidad del programa, es necesario que se adelanten esfuerzos para crear una cultura de calidad en toda la población estudiantil y se propone la realización de un estudio a nivel nacional que investigue estas percepciones en diferentes universidades en Colombia, teniendo como referente las implicaciones del contexto al que pertenecen.
\end{abstract}

Palabras clave: Calidad, Acreditación, Administración de Empresas, Percepción, Estudiantes.

\section{ABSTRACT}

This article aimed to explore the perceptions of students of Business Administration Program of the University of Magdalena on the accreditation process for high quality that is submitted to the Ministry of Education. For which a random sample of 100 students was taken, who are part of various academic semesters, answered a questionnaire that asked for their views on the process. The main results support the view that students have a huge sense of belonging to the program and believe

Fecha de recepción: Mayo 15 de 2015 / Fecha de aceptación: Julio 03 de 2015

Tipología: Artículo de Investigación Científica y Tecnológica

Para citar este artículo: Viloria, E. J., Bertel, N. M., \& Daza, C. A. (2015). Percepciones estudiantiles sobre el proceso de acreditación por alta calidad del Programa de Administración de Empresas de la Universidad del Magdalena. Praxis. Vol. 11, 89 - 102

\footnotetext{
*Administrador de Empresas. Universidad del Magdalena. Colombia.Email: javierviloriaescobar@gmail.com

**Especialista en Finanzas. Universidad del Magdalena. Colombia. Email: maripao.bertel@gmail.com

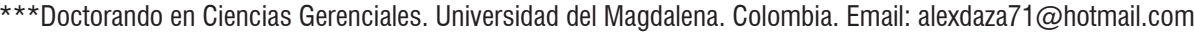


that it deserves accreditation by the Ministry and, further, this certificate becomes something positive for their careers. We conclude that, despite the apparent acceptance of the conditions of program quality, it is necessary that efforts be brought forward to create a culture of quality throughout the student population and a and a nationwide study is proposed to investigate these perceptions in various universities in Colombia, having as reference the implications of the context to which they belong.

Keywords: Quality, Accreditation, Management, Perception, Students.

\section{INTRODUCCIÓN}

U a Universidad del Magdalena, como universidad pública de carácter territorial, comprometida con el desarrollo de la autonomía y la excelencia académica, estableció desde el año 2009 como política y proceso estratégico, construir en el interior de sus Facultades y Programas Académicos una cultura de autoevaluación y mejoramiento continuo.

El Programa de Administración de Empresas, adscrito a la Facultad de Ciencias Empresariales y Económicas, tuvo sus inicios en la década de 1970 como Administración Agropecuaria, en la modalidad tecnológica y luego integrado a un ciclo profesional hacia finales de la década de 1980. Más adelante, en el año 1993 da el paso hacia un programa profesional de Administración de Empresas con 3 énfasis: Turístico, Agropecuario, Finanzas y Sistemas; desde entonces éste Programa ha trabajado por la comprensión de las organizaciones, el pensamiento administrativo y el quehacer del Administrador de Empresas, de cara a los desafíos propios de la globalización en el interior de las organizaciones del País y la Región Caribe.

Dentro de este marco, al obtener el Registro Calificado en el año 2005, la Universidad se propuso continuar fortaleciendo esta labor y como un paso más en la ruta de consolidar un programa de alta calidad, se decidió iniciar un proceso de autoevaluación entre los años 2009-2011 que posibilitó la renovación del mencionado Registro en el año 2012. En este mismo año se continuó con la autoevaluación, revisión y análisis de los de los distintos factores de calidad del Programa, que indicara la posibilidad de postular el mismo a evaluación de pares académicos externos con el fin de buscar la Acreditación de Alta Calidad del Programa.

En este sentido, la Facultad de Ciencias Empresariales y Económicas y el Programa de Administración de Empresas desarrollaron acciones de mejora, con el firme propósito de garantizar los estándares necesarios que evidenciaran la calidad del Programa y en consecuencia su presentación ante el Consejo Nacional de Acreditación (CNA), para iniciar el proceso que permita obtener la respectiva Acreditación.

La presente investigación tuvo como objetivo recopilar las percepciones que tienen los estudiantes del Programa de Administración de Empresas de la Universidad del Magdalena sobre el proceso de acreditación por alta calidad al que se sometió por parte del Consejo Nacional de Acreditación del Ministerio de Educación Nacional, con la intención de verificar socialmente el conocimiento que se tiene del proceso, las percepciones sobre la influencia de este en la formación profesional y desempeño laboral, y los niveles de participación de los estudiantes en las actividades de autoevaluación que se han desarrollado.

Inicialmente, la investigación delimita unos soportes teóricos relacionados con la calidad y los procesos de acreditación en la educación superior. Luego se aclara la metodología utilizada 
para el desarrollo del estudio y el procesamiento de la información recolectada para presentar los principales resultados encontrados en el marco del proceso investigativo en torno a las categorías definidas para los intereses de los autores y finalmente, se presentan las principales conclusiones y apreciaciones sobre el fenómeno y, se proponen, algunos escenarios investigativos para futuras intenciones por parte de la academia.

Para Gómez (2006) los procesos de globalización dentro de los cuales se desarrolla la educación, no solo se enfocan en generar conocimientos esenciales que permitan la formación de estudiantes en ciudadanos integrales con habilidades para la participación activa dentro de sus comunidades sino que "por el contrario, la educación global constituye un valor agregado, que impulsa el desarrollo del conocimiento en función de capacitar al individuo para crear riqueza y materializar su plan de vida” (p. 168). Desde esta perspectiva, la calidad con la que se ejecutan dichos procesos constituye un elemento de vital importancia para garantizar la satisfacción de los futuros egresados de los programas académicos, puesto que permiten configurar un escenario financiero que se relaciona con las expectativas de los productos.

Según Peresson (2007), para que en las organizaciones la calidad se convierta en una ventaja competitiva que le permita obtener privilegios en el mercado debe adoptarse por parte de todos los integrantes, esta calidad, como un estilo de vida. En este sentido, y teniendo como referencia las actuales políticas del Estado colombiano, que condicionan a las instituciones de educación superior y sus programas, para ser receptoras de recursos oficiales de la nación y para recibir estudiantes que hacen parte de programas especiales de los gobiernos, certificar la calidad, aunque siempre se ha considerado como un proceso voluntario, es casi un requisito.

La calidad, desde el punto de vista organizacional, se relaciona con el cumplimiento de requisitos y la satisfacción de necesidades (Sánchez, 2007; Crosby, 2000) cuyo significado está determinado por el contexto (Pérez, 2007), y su gestión es un instrumento clave para el mejoramiento de los resultados en las organizaciones (Pertusa, Tarí, Pereira, Molina \& López, 2013) que genera una cultura de planeación, ejecución, verificación e implementación de acciones correctivas (Sánchez, 2007). Por lo tanto, la gestión de la calidad influye positivamente en el desempeño organizacional (Camisón, Boronat, Villar \& Puig, 2009).

Pero el término de calidad en la educación goza de una relatividad basada en las expectativas que tienen las personas sobre los procesos y las experiencias que han vivido a lo largo de su vida. Para Pérez (2007) "la calidad es un término ambiguo que depende del concepto de educación que se tenga, y que se relaciona con el modelo de hombre y de sociedad que se pretenda” (p. 202). En este mismo sentido, Menin (2013) expresa que al hablar de educación de calidad se generan confrontaciones, debido a que cada persona expresa lo que se le ocurre, basada en sus ideologías y subjetividad. Quiroz (2007, citando a Ratcliff, 2005), sostiene que "lo que se ve y no se ve en cuanto a la calidad en un programa o institución es el resultado de la filosofía educativa, pensamientos, valores, normas y poder, porque la calidad es un constructo individual y social (...)” (p. 98).

Buendía (2007) aclara que "el concepto de calidad en la educación superior es una construcción que obedece a un contexto político, económico y social dinámico en el que están inmersos los actores de la educación superior” (p. 33). Debido a las necesidades que encontraron, las instituciones de educación superior de ser eficientes y desarrollar en sus programas la mejora continua, los actuales procesos de autoevaluación a los que se someten las instituciones de educación y sus programas académicos en Colombia para recibir certificación por parte del Ministerio de Educación son mecanismos que les permiten demostrar la calidad en los diferentes factores que los componen.

Conocedores de la importancia de estos procesos para garantizar el impacto de los profesionales en formación en la sociedad a través del planteamiento de estrategias eficientes para satisfacer las necesidades existentes, el Programa 
de Administración de Empresas de la Universidad del Magdalena inicia los trámites para conseguir una validación de la calidad de sus procesos académicos por parte del Consejo Nacional de Acreditación con grandes esfuerzos por autoreconocer, a través de diferentes estrategias de movilización de los actores sociales que lo integran, las condiciones actuales del servicio que ofrece y presentar una ponderación del mismo ante el organismo certificador.

Enfocando estas intenciones, y teniendo como referencia que la educación de calidad realiza transformaciones en los estudiantes a través de un enriquecimiento de sus habilidades y el otorgamiento de poder para la toma de decisiones a partir de la construcción de valor agregado relacionado con un componente pedagógico y educativo (Clemenza, Ferrer \& Pelekais, 2005), el Programa lo que busca es certificar la formación de profesionales integrales con amplias capacidades para la transformación social de los contextos en los cuales intervengan, luego de someterse a un estado de reflexión sobre el grado de cumplimiento de este objetivo.

Este proceso de autoevaluación genera un conjunto de apreciaciones ponderadas sobre las condiciones de calidad en diferentes factores evaluados por el Ministerio de Educación, pero no indaga a profundidad las percepciones que tienen los diferentes estamentos universitarios sobre el Proceso de Acreditación de Alta Calidad. En este sentido, la presente investigación pretende hacer una aproximación a la relevancia que representa para los estudiantes del programa el certificado de calidad del Ministerio y sus posibles repercusiones en su formación académica y el ejercicio profesional. Además, se pretende validar el grado de conocimiento sobre un proceso que se ha convertido una necesidad para los programas e instituciones de educación superior en el país. Para Buendía, Sampredo y Acosta (2013) muchos estudios han demostrado los efectos positivos de la evaluación y acreditación para el mejoramiento de la calidad. Sin embargo, carecen de una recopilación de percepciones de los alumnos, como actores principales, sobre los sistemas de información de los trabajos realizados sobre las entidades evaluadoras y certificadoras, y los resultados que se obtienen. Mientras que Ratcliff (2005) sostiene que la calidad en la educación es un constructo desde una consideración individual y social que se pone en acción con el desarrollo del currículo y tiene en cuenta al estudiante desde diferentes perspectivas.

Fresán (2007), referenciando a Aguerrondo (2003) sostiene que un parámetro para medir "la calidad de un sistema educativo es su capacidad de alimentar al sistema cultural con los valores que éste reclama para constituirse como sociedad, es decir, si cumple con su función de ayudar a la integración social” (p. 55). Y Londoño y Ramírez (2012) expresan que la calidad se verá reflejada en la formación de profesionales idóneos que respondan a las necesidades de la sociedad y contribuyan a su transformación. Desde esta perspectiva, la calidad de un programa educativo está determinada en gran manera por el impacto de sus graduados en los sistemas sociales donde se insertan para cumplir su misión profesional.

Mientras que para Clemenza et al., (2005) la calidad en la educación superior es un factor determinante de competitividad. Es precisamente por estas consideraciones que las universidades deciden someter a evaluación sus procesos institucionales y sus programas de formación. Buscando una mayor pertinencia en los contextos y mejorar sus niveles de productividad y satisfacción de clientes. Urbano (2007) cree que la acreditación pretende fortalecer y legitimar las instituciones y sus programas académicos y para tal efecto, el sistema del CNA adopta dos interpretaciones de calidad: "La primera de ellas apunta a la calidad entendida como excepción, esto es, a la excelencia en relación con estándares y a la reputación de los centros en función de sus medios y recursos" (Hernández, (2002, referenciado por Urbano, 2007, p. 145). Mientras que la segunda "hace referencia a la calidad como adecuación a propósitos Hernández (2002) o como pertinencia respecto del propósito (Martin \& Rouhiainen, 2003)" (Urbano, 2007, p. 145). La acreditación es un proceso fundamental para las instituciones de educación superior al involucrar, según Sánchez (2013) a todos los actores sociales que las integran y llevar implícita la 
comprobación de los estándares de calidad de la estructura, procesos y resultados en correspondencia con su objetivo social y disposiciones estatales.

En otro sentido, y con la intención de soportar la importancia del estudio de las percepciones en las ciencias sociales, Calixto y Herrera (2010) exponen que surgen de la necesidad que se posee para el desenvolvimiento en un entorno determinado y hacer frente a los problemas de la sociedad. Mientras que para Corbella (1994), con la percepción el sujeto obtiene de manera automática e inconsciente la información del medio ambiente y para Pidgeon (1998), la percepción determina juicios, decisiones y conductas, y conlleva a acciones con consecuencias reales.

La percepción ofrece los insumos sobre los cuales se forman las evidencias de acuerdo a los significados que se expresan en la sociedad y permite determinar las cualidades y elementos cualitativos de los objetos y la realidad. Esta característica de los grupos sociales permite una menor interpretación de los fenómenos organizaciones desde las categorías conscientes y subconscientes del sujeto (Vargas, 1994).

Dese estos aportes, se sustenta la intención de estudiar y recopilar las percepciones sobre el proceso de acreditación del Programa de Administración de Empresas por parte de los estudiantes.

En este sentido, se valoran los aportes que relacionan la calidad en la educación con los niveles de competitividad de la misma, en la medida que permite entender y soporta todos los esfuerzos en la autoevaluación por un objetivo específico para las instituciones educativas. Pero también, y es lo que se prima en este estudio, se rescata el componente social que configuran los procesos de acreditación, porque más allá de todas las posiciones teóricas que se puedan desarrollar, es claro que estos son una dinámica de movilización social de los actores implicados, que responden a unos criterios definidos en los lineamientos de los organismos estatales pero que no reflejan, las interpretaciones y significados que tienen para la comunidad estudiantil, las implicaciones y repercusiones en su formación profesional y futuro desempeño laboral la acreditación de alta calidad de un programa.

\section{METODOLOGÍA}

Esta investigación es de carácter exploratorio con un modelo descriptivo mixto que incluye al aspecto tanto cuantitativo como cualitativo. La decisión del estudio se toma considerando las implicaciones sociales que expresan los procesos de acreditación en programas académicos en las universidades. El estudio se realizó en la Universidad del Magdalena ubicada en la ciudad de Santa Marta durante el segundo semestre del año 2014, tiene como referente metodológico el estudio desarrollado por Buendía, Sampredo y Acosta (2013) que intentaban analizar, desde la perspectiva de los estudiantes, los procesos de evaluación y acreditación de los programas de licenciatura en México.

\section{Población y Muestra}

La población está definida por los estudiantes del Programa de Administración de Empresas que para el periodo estaba constituida por 1.087 estudiantes, y considerando los intereses exploratorios de la investigación se seleccionó una muestra aleatoria de 100 personas entre los diferentes semestres académicos para aplicar el cuestionario.

\section{Instrumentos}

En la investigación se empleó un cuestionario elaborado por los autores y teniendo como referencia las necesidades de información se establecieron las siguientes variables a evaluar: conocimiento de los procesos de Acreditación del Programa (que se mide en tres interrogantes); percepción sobre las condiciones de calidad del programa (evaluado a través de tres preguntas); y participación en las actividades de autoevaluación (para la medición de la cual se aplicaron dos preguntas). Adicionalmente, se colocó un interrogante para que los individuos pudieran desarrollar de forma abierta su opinión sobre el proceso de acreditación del programa. 


\section{Procedimiento}

La investigación se dividió en las etapas que se describen a continuación:

Primera: permitió una consulta teórica sobre los conceptos que se han desarrollado sobre calidad con el objeto de establecer una contextualización conceptual sobre la problemática de análisis y tener claros los antecedentes sobre las investigaciones en este campo.

Segunda: en esta etapa se procedió a elaborar el instrumento para la recolección de la información de la muestra seleccionada.

Tercera: durante esta etapa se realizó la investigación de campo en las instalaciones de la sede principal de la Universidad del Magdalena durante el segundo semestre del 2014. Los cuestionarios se entregaron en forma física a 100 estudiantes del programa de Administración de Empresas. El $66,00 \%$ de los encuestados pertenecen al rango académico entre primer y quinto semestre, mientras que el $34 \%$ restante de la muestra oscilan entre sexto semestre y estudiante en condición de grado. Los criterios para elegir las representaciones de la muestra por semestres académicos se relacionan con que la mayor cantidad de la población de estudiantes del Programa se encuentran entre los primeros cinco periodos académicos. Lo que permite tener una mejor interpretación de las representaciones sociales de la muestra en relación con la cantidad y características de los estudiantes. La totalidad de los cuestionarios fueron resueltos por los participantes.

Cuarta: el procesamiento de los datos recolectados en los cuestionarios se realizó mediante el software STATGRAPHICS, para la organización de la información cuantitativa, mientras que para la información cualitativa recolectada en el test a partir de la pregunta abierta sobre su opinión del proceso se hizo una transcripción a documentos en Word enumerando cada respuesta con un número del cuestionario entregado.

Quinta: el análisis de los datos se realizó teniendo como referente la información consolidada en los siguientes cuadros.

La tabla 1 presenta los valores mínimos y máximos que se pueden otorgar en la calificación por cada variable, así como el número de preguntas que contiene cada una y los puntajes que se pueden lograr al sumar las calificaciones. Además, se presenta el rango de valores máximos y mínimos que se puede conseguir en cada cuestionario al completar la sumatoria de los puntajes entregados por variable.

En la investigación se establecieron preguntas de cinco tipos. Las de tipo uno tienen preguntas de carácter sí o no; las de tipo dos consideran las opciones de respuesta excelente, bueno, regular y malo; las de tercer tipo tienen las posibilidades de contestación positiva o negativa; las de tipo cuatro permiten elegir entre mucho, poco, muy poco y no ha participado/ no revisa; y las de quinto tipo que se establecen abiertas para presentar opiniones. La tabla presenta las discriminaciones de tipos de preguntas incluidos en el cuestionario por variable. 
Tabla 1

Valores de las variables del cuestionario

\begin{tabular}{|c|c|c|c|c|c|}
\hline \multirow{2}{*}{ Variable } & \multicolumn{2}{|c|}{ Calificación } & \multirow{2}{*}{$\begin{array}{c}\text { No. } \\
\text { Preguntas }\end{array}$} & \multicolumn{2}{|c|}{ Puntaje por variable } \\
\hline & Mínimo & Máximo & & Mínimo & Máximo \\
\hline Conocimiento & 0 & 1 & 3 & 0 & 3 \\
\hline Percepción & 0 & 3 & 3 & 0 & 5 \\
\hline Participación & 0 & 3 & 2 & 0 & 6 \\
\hline \multicolumn{5}{|c|}{ Puntaje Máximo por encuesta } & $\begin{array}{c}14 \\
\text { puntos }\end{array}$ \\
\hline \multicolumn{5}{|c|}{ Puntaje Mínimo por encuesta } & 0 puntos \\
\hline
\end{tabular}

Fuente: elaboración propia de los autores.

Tabla 2

Tipología de respuestas por variables

\begin{tabular}{|l|c|c|}
\hline \multicolumn{1}{|c|}{ Variable } & Preguntas & Respuestas \\
\hline Conocimiento & 3 & Tipo 1 \\
\hline Percepción & 3 & Tipo $1 / 2 / 3$ \\
\hline Participación & 2 & Tipo 4 \\
\hline Opinión Del Proceso & 1 & Tipo 5 \\
\hline
\end{tabular}

Fuente: elaboración propia de los autores.

\section{RESULTADOS}

Los estudiantes encuestados de los semestres inferiores (del primero al quinto) representaron el $66,00 \%$ de los datos recolectados, la mayor población de encuestados se concentró en el cuarto semestre con un total de 22 cuestionarios del total de la muestra seleccionada. La figura 1, muestra la relación de conocimiento de los estudiantes del programa sobre el proceso de Acreditación, en donde 56 de 100 encuestados poseen un 100\% conocimiento del proceso de Acreditación, mientras que 4/100 tiene un conocimiento nulo $(0,00 \%)$. En promedio el $81,44 \%$ de la población manifiesta tener un conocimiento del proceso de acreditación, dado que la desviación estándar se ubica en 0,2516 indicando un comportamiento positivo de la medición de la variable de conocimiento dado que refleja un interés en el proceso por parte de los diferentes semestres del programa.

Los datos específicos sobre las preguntas ejecutadas en esta variable permiten decir que solo el $8 \%$ de los estudiantes no entienden qué significa un proceso de Acreditación de Alta Calidad, mientras que el 92\% restante tiene alguna idea de las significaciones del proceso. El $40 \%$ no conoce los requerimientos para que el programa sea acreditado por parte del Ministerio de Educación y solo un $8 \%$ manifiesta no saber que el Programa de Administración de Empresas de la Universidad del Magdalena se encuentra en este proceso. 


\section{Figura 1}

Medición Variable Conocimiento

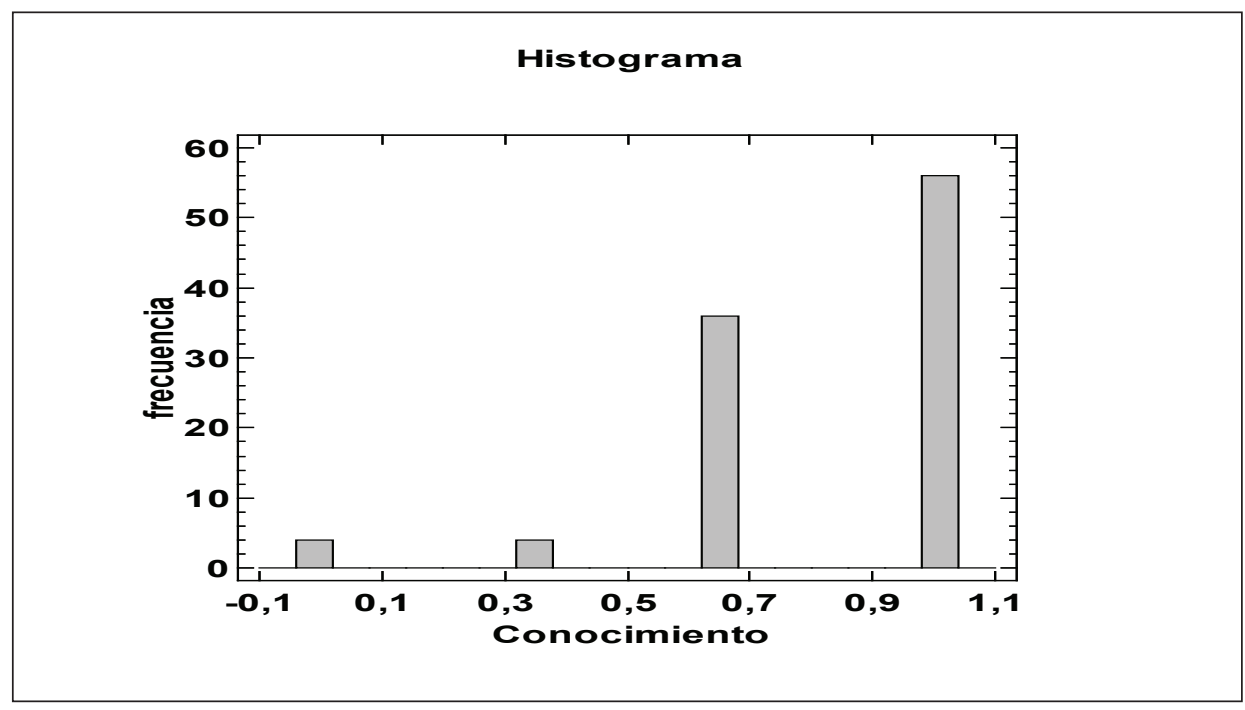

Fuente: elaboración propia de los autores.

Por su parte, la variable percepción en la calidad del programa presenta un comportamiento similar a la variable conocimiento de los procesos de acreditación, dado que los estudiantes encuestados señalan tener una percepción positiva del proceso de acreditación y la calidad del programa evaluándolo en términos generales como bueno, con beneficios para su formación profesional y considerando que debe ser otorgada la Acreditación al programa, esto se refleja en que $3 / 100$ encuestados calificaron de manera negativa (menos o superior al 40\%), mientras que el 54/100 asignaron una puntuación de $80 \%$ para la Acreditación del programa. Siendo el promedio de valoración de la percepción del 85,8\% y una desviación estándar del 0,1372.

Desde el análisis de las preguntas que integran la variable, el 7\% de las personas evalúan el proceso de acreditación del programa como regular, el $54 \%$ lo consideran bueno mientras que el $39 \%$ lo entienden como excelente. A la pregunta acerca de si el programa se merece la Acreditación solo el $3 \%$ de la muestra considera que no la merece.

La variable de participación en las actividades de autoevaluación del programa muestra una representación múltiple del nivel de vinculación donde solo 9/100 de los encuestados presenta una participación del 100\% mientras que 5/100 de los estudiantes que respondieron el cuestionario manifestaron tener una participación nula $(0,00)$ por lo que la mayor concentración de la población se ubica en promedio en un 58,32\% de participación en actividades de autoevaluación (talleres, diálogos, encuestas, etc.) o en la revisión de los comunicados del proceso, siendo los porcentajes mayores porcentajes de participación el $67 \%$ que se ubican por encima del 25 /100 encuestados y el $83 \%$ que sobrepasan el 20/100. 


\section{Figura 2}

Medición Variable Percepción

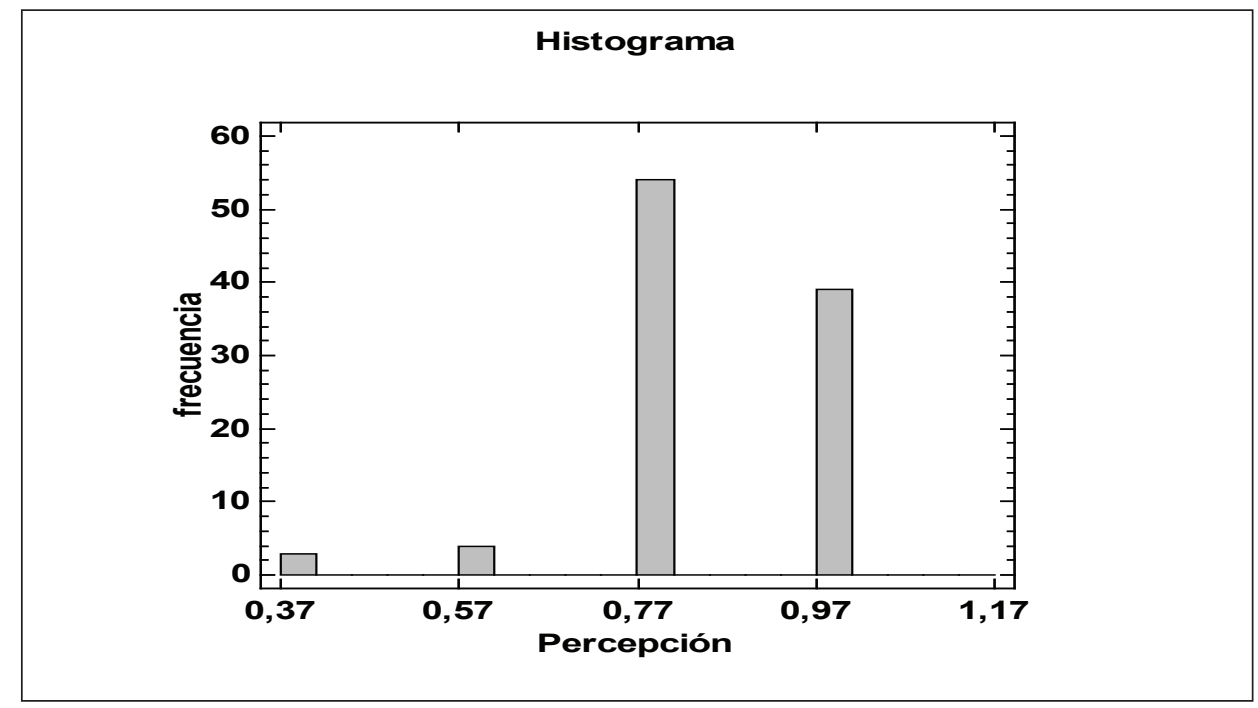

Fuente: elaboración propia de los autores.

\section{Figura 3}

Medición Variable Participación

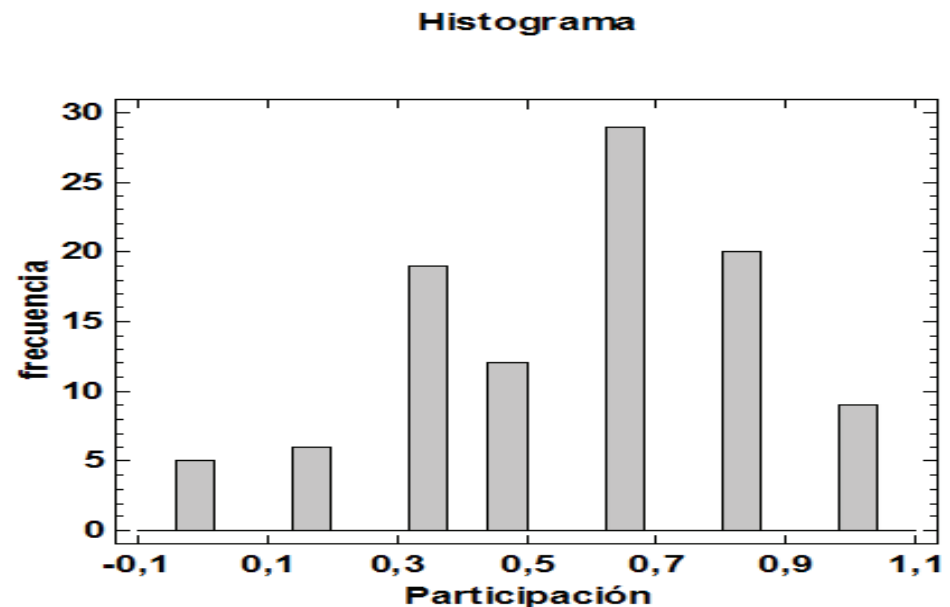

Fuente: elaboración propia de los autores. 
El nivel de participación de los estudiantes se presenta en la figura 4.

\section{Figura 4}

Nivel de participación en actividades de autoevaluación

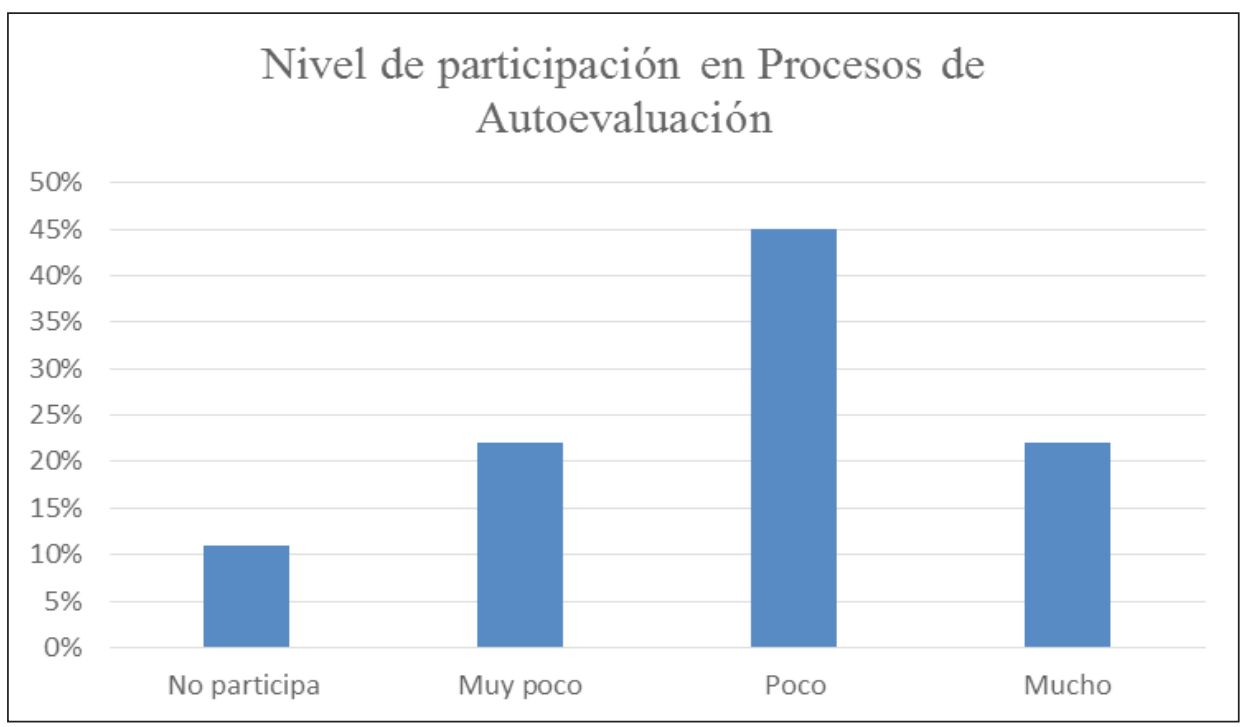

Fuente: elaboración propia de los autores.

Dentro de la variable de participación se mide la frecuencia con que los estudiantes revisan los diferentes informes que se generan en el Programa sobre los procesos de autoevaluación y se presentan al respecto los datos en la figura 5.

\section{Figura 5}

Nivel de revisión de informes de autoevaluación

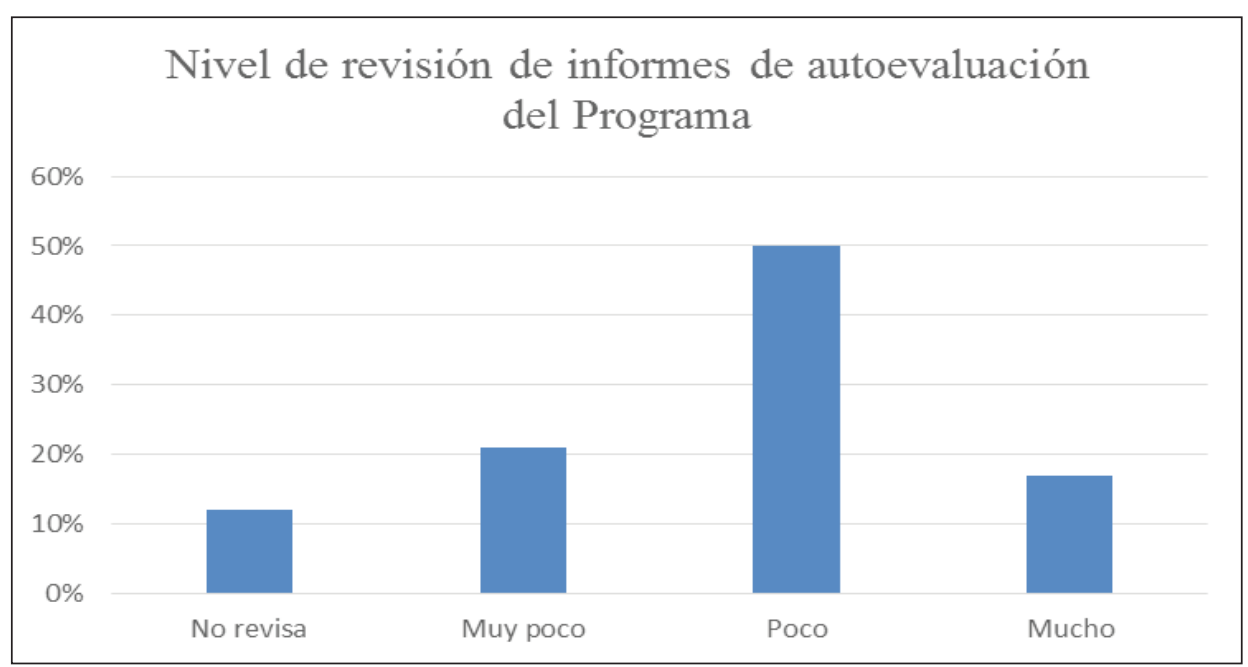

Fuente: elaboración propia de los autores. 
En la evaluación general de los componentes (variables) del cuestionario se señala que el $73,09 \%$ de los encuestados presentan un alto sentido de pertenencia con el Programa de Administración de Empresas con relación al proceso de Acreditación de Alta Calidad, debido a que en los diferentes semestres las valoraciones totales del cuestionario con respecto a la suma de las variables Conocimiento, Percepción y Participación muestran una variedad de resultados que comprenden el intervalo de $70 \%$ al $100 \%$ del sentido de pertenencia de los estudiantes, siendo el mínimo del 0,36\% obtenido en 4 de 100 cuestionarios.

Los conocimientos, percepciones y participaciones de los estudiantes en torno al proceso de certificación de calidad del programa permiten configurar un escenario favorable desde este estamento en torno a las implicaciones positivas y compromisos que tienen los actores para construir una entidad colectiva que respalde las dimensiones establecidas en los documentos oficiales resultantes de los procesos de autoevaluación. Además, valida las posiciones teóricas de diversos autores que consideran la calidad y los procesos de acreditación como elementos que permiten que las instituciones y los programas académicos tengan un mayor impacto en la sociedad a partir de la formación de profesionales integrales con mayores habilidades para entender las necesidades del entorno y crear herramientas para atenderlas.

Como una manera de medir las opiniones y tener un mayor acercamiento a las percepciones de los estudiantes sobre el proceso de acreditación, a continuación se presentan algunas voces con el propósito de configurar los diálogos que se manejan en el estamento estudiantil y presentar, desde una perspectiva social, las connotaciones y significaciones que tienen para los estudiantes, como actores activos de los procesos de gestión relacionados con la calidad, los adelantos que se han registrado en el proceso de acreditación del programa y sus posibles repercusiones para el establecimiento de sus vidas profesionales.

\section{Tabla 3}

Voces de los estudiantes sobre el Proceso de Acreditación

\begin{tabular}{|c|l|c|}
\hline Cuestionario & \multicolumn{1}{|c|}{ Percepción } & Semestre \\
\hline \multirow{3}{*}{ C7 } & $\begin{array}{l}\text { Al lograr la acreditación del programa se crean } \\
\text { ventajas competitivas para la carrera y la uni- } \\
\text { versidad frente a las demás existentes en el país } \\
\text { que aún no lo han conseguido y significa estar } \\
\text { al mismo nivel de las mejores universidades, } \\
\text { es un sello valioso que brinda credibilidad a } \\
\text { nuestros conocimientos. }\end{array}$ & \\
\hline C15 & $\begin{array}{l}\text { El proceso de acreditación es acertado porque } \\
\text { nos permite mejorar nuestras oportunidades } \\
\text { laborales pero aún existen grandes retos que } \\
\text { debemos superar para lograrlo. }\end{array}$ & Cuarto semestre \\
\hline
\end{tabular}




\begin{tabular}{|c|l|c|}
\hline Cuestionario & \multicolumn{1}{|c|}{ Percepción } & Semestre \\
\hline C27 & $\begin{array}{l}\text { Es importante ampliar la planta docente } \\
\text { del Programa para lograr el objetivo de la } \\
\text { Acreditación. }\end{array}$ & Tercer semestre \\
\hline C29 & $\begin{array}{l}\text { La Acreditación por Alta Calidad del Programa } \\
\text { de Administración de Empresas nos permite, } \\
\text { como futuros profesionales, mejores posibilida- } \\
\text { des de acceso al campo laboral y garantiza la } \\
\text { formación de personas integrales. }\end{array}$ & Estudiante de \\
\hline C55 & $\begin{array}{l}\text { La calidad certificada del Programa nos permite } \\
\text { una mejor formación como administradores. }\end{array}$ & Segundo \\
semestre
\end{tabular}

Fuente: elaboración propia de los autores.

Desde las interpretaciones estudiantiles y teniendo como referencia los soportes teóricos expuestos en la investigación, se puede resaltar que a pesar de que existe un criterio casi general de la acreditación como un soporte positivo para las estudiantes, este es relativo a las condiciones y conocimientos de cada actor involucrado. Al entenderse la calidad como un término relativo y subjetivo, así se comprenden las repercusiones del proceso sobre los participantes.

\section{CONCLUSIÓN Y DISCUSIÓN}

En el marco de los actuales procesos de autoevaluación que se desarrollan al interior de las instituciones de educación superior en Colombia y en los programas académicos que ofertan con el objeto de lograr la Acreditación de Alta Calidad que otorga el Ministerio de Educación Nacional, la representación 
social entendida como un constructo del conocimiento, percepción y participación de los estamentos institucionales se constituye en un soporte para validar los contenidos técnicos de las condiciones de calidad registradas en los informes oficiales. En este sentido, la configuración del proceso a través de las perspectivas sociales es un instrumento que demuestra la identificación y adaptación a una cultura de calidad por parte de toda la comunidad universitaria.

El estudio exploratorio que se ha implementado permite mencionar que los estudiantes de Administración de Empresas de la Universidad de la Universidad del Magdalena han entendido esta dinámica y reconocen como positivo el proceso de acreditación del programa. Razón por la cual, tienen un alto grado de conocimiento sobre la gestión institucional que se desarrolla para tal fin, tienen percepciones positivas sobre los efectos de la certificación para su vida profesional y en gran medida, han participado en los diferentes escenarios que se generan para la autoevaluación de las condiciones de calidad del programa. En este sentido, se puede arriesgar la premisa de que el programa ya cuenta con una acreditación social de sus procesos y solo se requiere que el Ministerio valide la construcción social que se ha forjado al respecto. Aunque se recomienda seguir trabajando por lograr una mayor movilización social en torno al proceso y poder acoger en torno a estas dimensiones que vive la comunidad académica y administrativa del programa a los estudiantes que tienen menores niveles de conocimiento y participación de esta dinámica universitaria.

En términos de dimensiones instituidas e instituyentes, es claro que existe una gran aproximación entre los documentos establecidos formalmente desde los resultados del proceso de autoevaluación y cumplimiento de requisitos del programa, con los imaginarios sociales que se construyen, al menos desde el estamento estudiantil, sobre las significaciones y percepciones del proceso.

Esta investigación contribuye a crear una mirada social del proceso de acreditación del programa y genera insumos para medir el impacto tiene dentro de la comunidad estudiantil desde un enfoque exploratorio. Lo que permite tener una base para la implementación de un proyecto de investigación a profundidad que mida las variables descritas en la metodología en una muestra más amplia y considere el análisis de nuevos factores relacionados con el proceso. Además, es indispensable indagar por estas consideraciones sociales en cada uno de los programas de la Universidad del Magdalena que se están sometiendo a una evaluación por parte del Consejo Nacional de Acreditación.

Se propone, en un espacio más amplio, la ejecución de un proyecto investigativo en red que analice las percepciones de los diferentes estamentos universitarios en programas similares de diferentes universidades colombianas desde una perspectiva del contexto regional, con el propósito de lograr un mapa social sobre el proceso de acreditación en Colombia y levantar una voz conjunta que le permita al Ministerio de Educación Nacional tener un insumo para tomar decisiones en torno al mejoramiento del proceso y los sistemas e indicadores de evaluación que se han implementado en los últimos años.

\section{REFERENCIAS BIBLIOGRÁFICAS}

Buendía, A. (2007). El concepto de calidad: una construcción en la educación superior. Reencuentro, 50, 28-34.

Buendía, A., Sampedro, J., \& Acosta, A. (2013). ¿La evaluación y acreditación mejoran la calidad de los programas de Licenciatura en México? La otra mirada, los estudiantes. El caso de seis universidades mexicanas. Reencuentro, 58-68.

Calixto, R., \& Herrera, L. (2010). Estudios sobre las percepciones y la educación ambiental. Tiempo de Educar, 11 (22), 227-249.

Camisón, C., Boronat, M., Villar, A., \& Puig, A. (2009). Sistemas de gestión de la calidad y desempeño: importancia de las prácticas de gestión del conocimiento y de I + D. Revista Europea de Dirección y Economía de la Empresa, 18 (1), 123-134.

Clemenza, C., Ferrer, J., \& Pelekais, C. (2005). La calidad como elemento competitivo en las Universidades. Revista de Artes y Humanidades UNICA, 6 (14), 55-83.

Corbella, R. (1994). Descubrir la Psicología. Percepción. Barcelona: Editorial Folio.

Crosby, P. (2000). Calidad sin lágrimas. México D.F.: Grupo Patria Cultural S.A. de C.V. 
Fresán, M. (2007). Repensar la calidad en la educación superior en el nuevo milenio. Reencuentro, 50, 52-59.

Gómez, J. (2006). Calidad de la educación Universitaria. Ingeniare. Revista Chilena de Ingeniería, 14 (3), 168-169.

Londoño, J., \& Ramírez, D. (2012). Cultura de la autoevaluación: cimiento para la acreditación de programas académicos en modalidad virtual. Revista Virtual Universidad Católica del Norte, 36, 1-6.

Menin, O. (2013). ¿Qué es una educación de calidad? Praxis Educativa (Arg), 17 (1), 24-27.

Peresson, L. (2007). Sistemas de Gestión de la Calidad con enfoque al cliente. España: Universidad de Valladolid.

Pérez, A. (2007). Calidad de la educación popular. Educere, 11 (37), 201-208.

Pertusa, E., Tarí, J., Pereira, J., Molina, J., \& López, M. (2013). Certificación en calidad, resultados empresariales y estructura organizativa en el sector hotelero español. Intangible Capital, 9 (1), 199-224.

Pidgeon, N. (1998). Risk assessment, risk values and the social science programme: why we do need risk perception research. Reliab Engineering Syst Safety, 59, 5-15.

Quiroz, E. (2007). Competencias profesionales y calidad en la educación superior. Reencuentro, 50, 93-99.
Ratcliff, J. (2005). Dynamic and communicative aspects of quaility Assurance. En W. De Vries, Calidad, eficiencia y evaluación de la educación superior. España: Riseu/Netbiblo.

Romero, N. (2007). Gerencia para transformar las universidades públicas autónomas de Venezuela. Revista Venezolana de Gerencia, 12 (40), 599-621.

Sánchez, J. (2007). Administración y Certificación de la Calidad mediante la Norma ISO 9001:2000. Bogotá: Gente Nueva.

Sánchez, R. (2013). Acreditación académica institucional. Archivo Médico de Camagüey, 17 (6), 641-647.

Urbano, N. (2007). Efectos de la implementación del modelo colombiano de acreditación de programas académicos. Un análisis a partir del caso de los programas tecnológicos. Universitas Humanística, 64, 139-161.

Vargas, L. (1994). Sobre el concepto de percepción. Alteridades, 4 (8), 47-53.

Vergara, C., \& Aguirre, C. (2013). Sistema de aseguramiento de la calidad académica en programas de postgrado. Proceso de autoevaluación - Estudio de caso. En A. Beltrán, C. Cobo, E. Chacón, \& C. Restrepo, La Administración frente a los desafíos de la economía global (págs. 304-324). Cali: Universidad del Valle. 